\title{
INFLUENCE DE L'ECOSYSTEME ET DU STADE DE DEVELOPPEMENT DES PANICULES SUR L'ANDROGENESE CHEZ LE RIZ
}

\author{
M. DOBO ${ }^{1}$, N. K. YAO ${ }^{2}$ et J. P. MONTY 3 \\ ${ }^{1}$ Département de Génétique, Université d'Abobo-Adjamé, URES Daloa, BP 150, Daloa, Côte d'Ivoire. \\ E-mail : dmccaire2006@yahoo.com \\ ${ }^{2}$ Laboratoire de Biotechnologie, Africa Rice Center, BP 01 BP 2031Cotonou, Benin \\ 3FFARA PMB CT 173. Accra, Ghana. mjones@fara-africa.org
}

\begin{abstract}
RESUME
L'étude vise à déterminer l'écosystème et le stade de panicule favorables à l'androgenèse chez le riz sur le site d'<<Africa Rice>> (ex-ADRAO) à M'Bé, Bouaké. Le matériel végétal a consisté en deux variétés de Oryza glaberrima (CG 14 et CG 20) et 3 de Oryza sativa de type japonica (WAB 56-50 et TAIPEI 309) et indica (BOUAKE 189). Les cals induites à partir d'anthères sur le milieu N6 modifié contenant $0,5 \mathrm{mg} \mathrm{l}^{-1}$ de kinétine, $2,5 \mathrm{mg} \mathrm{l}^{-1}$ de dichlorophenoxy acétique acide (2,4-D), et $15 \%$ de lait de coco, sont ensuite régénérés sur milieu MS additionné de $1 \mathrm{mg} \mathrm{l}^{-1} \mathrm{~d}^{\prime}$ acide -naphtalène acétique (ANA) et $4 \mathrm{mg} \mathrm{l}^{-1}$ kinétine. Les résultats ont révélé une forte interaction génotype-écosystème. En effet, chaque génotype a eu une bonne réponse en androgenèse avec les anthères provenant de son écosystème habituel de croissance. Ainsi, les glaberrima et les japonica se sont bien comportés en androgenèse, au niveau des plateaux et l'indica dans les bas-fonds. Le stade jeune de la panicule (distance auriculaire entre 7 et 8,8 cm) a été le meilleur stade de prélèvement. Tous stades de panicule et écosystèmes confondus, les meilleurs résultats pour la production de cals et la régénération de plantules a été obtenue avec les sativa comparativement aux glaberrima.
\end{abstract}

Mots clés : Glaberrima, anthères, androgenèse, riz, ANA, 2,4-D.

\author{
ABSTRACT \\ INFLUENCE OF THE ECOSYSTEM AND PANICLE STAGE ON RICE ANDROGENESIS
}

The study aims to identify the ecosystem and panicle stage suited for rice anther culture at "Africa Rice" (previously WARDA) site located at M'Bé, Bouaké. Plant material consisted in two varieties of Oryza glaberrima (CG 14 and CG 20) and 3 of Oryza sativa, including two japonica (WAB 56-50 and TAIPEI 309) and one indica (BOUAKE 189). The callus were obtained on a modified N6 medium containing $0.5 \mathrm{mg} \mathrm{l}^{-1}$ of kinetin, $2.5 \mathrm{mg} \mathrm{l}^{-1}$ of 2,4-dichlorophenoxyacetic acid (2.4-D), and $15 \%$ of coconut milk, then regenerated on MS medium supplemented with one $\mathrm{mg} \mathrm{l}^{-1} \alpha$-naphtalenacetic acid (ANA) and $4 \mathrm{mg} \mathrm{l}^{-1}$ kinetin. The results revealed a strong interaction genotype-ecosystem. Indeed, the best performance in androgenesis for each genotype was obtained with anthers that originated from the ecosystem of natural occurring. Thus, glaberrima and japonica performed well in upland and the indica in lowland. The youngest stage of panicle (auricular distance of 7 to $8.8 \mathrm{~cm}$ ) was the best stage for androgenesis of these varieties. For all the panicle stages and ecosystems, sativa varieties gave the best responses both for callus production and plant regeneration than glaberrima varieties.

Key words : Glaberrima, sativa, anther, androgenesis, rice, ANA, 2,4-D. 


\section{INTRODUCTION}

L'ADRAO a entrepris de développer, par la méthode conventionnelle d'hybridation, des variétés interspécifiques à haut rendement combinant les caractères intéressants des deux espèces de riz cultivé Oryza sativa (originaire d'Asie) et Oryza glaberrima (originaire d'Afrique), ceci en vue de générer de nouvelles lignées homozygotes à haut rendement pouvant assurer des récoltes stables en dépit des conditions fluctuantes de l'environnement. Les nouvelles lignées issues de ces croisements cumulent le haut potentiel de rendement de $O$. sativa et la bonne tolérance aux stress biotiques (tolérance aux maladies, insectes, etc.) et abiotiques (sécheresse, acidités des sols, etc.) de $O$. glaberrima (Monty et al., 1997).

Mais, cette méthode est souvent limitée dans son application, car elle exige un long délai (Angladette, 1966) pour l'obtention des lignées fixées. De plus, les croisements de $O$. sativa avec $O$. glaberrima conduisent, dans de nombreux cas, à des plantes F1 portant des panicules totalement stériles (Monty et al., 1997). Pour faire face au problème de stérilité, I'on associe, à la méthode traditionnelle de sélection, d'autres techniques dont la culture in vitro et, particulièrement, celle d'anthères in vitro ou androgenèse. Cette méthode a pour avantage d'utiliser les fleurs mâles (anthères) et d'obtenir, grâce à l'haplodiploïdisation spontanée ou induite, des lignées fixées en une seule génération, contrairement à la sélection classique qui, nécessite au minimum, 7 générations et qui, de plus, ne permet pas l'expression des caractères récessifs.

La production de plantes haploïdes de riz $(O$. sativa) par culture d'anthères a été effectuée pour la première fois, par Niizeki et Oono (1968). D'autres travaux ont suivi avec toutes les sous espèces de $O$. sativa que sont les types japonica, javanica et indica. Des cas de cultures réussies ont également été publiés chez différents hybrides issus des croisements des sous groupes de O. sativa (Quimio et Zapata, 1990 ; Faruque et al., 2004). Quelques rares cas de succès ont été rapportés chez $O$. glaberrima par Woo et Wong (1983) et Guèye et Ndir (2010). Ainsi, grâce à l'andro-genèse, de nouvelles variétés de riz $O$. sativa ont été obtenues dans certains pays tels la Corée (Chung, 1992) et la Chine (Zhenhua, 1992).
Toutefois, de nombreuses études ont révélé que la conjonction d'un certain nombre de facteurs influence, de façon significative, l'androgenèse. Il a été rapporté que chez $O$. sativa, la sous espèce japonica répondait mieux à la culture d'anthères qu'indica et javanica (Shen et al., 1982 ; Faruque et al., 2004). Le stade de développement des panicules approprié à l'androgenèse chez ces sous espèces a varié d'une variété à une autre (Jähne et Lörz, 1995). Des études additionnelles ont démontré que l'androgenèse avait une base génétique avec effet additif des gènes et un effet maternel (Yan et al., 1996 ; Bagheri et Jelodar, 2008). Un certains nombre de prétraitements des panicules se sont avérés stimulateurs de l'androgenèse. Le plus courant est le choc thermique qui consiste à conserver des panicules à basses températures (4 à $13^{\circ} \mathrm{C}$ ) pendant au moins $10 \mathrm{j}$ (Genovesi et Magill, 1979).

De nombreuses études ont montré que les milieux de base d'induction de cals, à partir des anthères sont aussi déterminants pour une bonne androgenèse. Par exemple, le milieu N6 de Chu et al. (1975) s'est révélé convenable au type japonica, et le milieu He5 de Huang et al. (1978) au type indica. Les phytohormones ajoutées à ces milieux, en callogenèse sont génotypedépendant, toutefois les plus utilisées sont le 2,4-D et l'ANA (Cornejo-Martin et Primo-Millo, 1981). Des travaux ont révélé que des composés organiques tel que l'acide abscissique (Torrizo et Zapata, 1986), et certains acides aminés (Constance et al., 1993) apportés aux milieux stimulent l'androgenèse.

Par ailleurs, d'autres études ont montré que le milieu de croissance des plantes mères était aussi important en androgenèse. En effet, Zhenhua (1992) a montré que les plantes cultivées sur un sol de bonne fertilité donnent les meilleures réponses en culture d'anthères. Ceux de Zapata et al. (1985) signalent que la réponse en androgenèse du riz a varié en fonction des saisons ; la saison sèche étant plus favorable que la saison pluvieuse.

Cependant, malgré l'importante littérature traitant des conditions optimales de l'androgenèse chez le riz, aucune n'a clarifié l'influence de l'écosystème dans lequel évolue les plantesmères. Le site d'<<AfricaRice >> (ex-ADRAO) de M'Bé, Bouaké, est constitué de 3 écosystèmes, le plateau, l'hydromorphe et le 
bas-fond. L'ensemble de ces écosystèmes étant désigné sous le nom de $<<$ continuum $>>$ (Figure 1). L'objectif de ce travail est donc de déterminer l'écosystème le plus favorable et le stade de developpement de panicule approprié, pour l'androgenèse chez le rizsur ce site d'«Africa Rice».

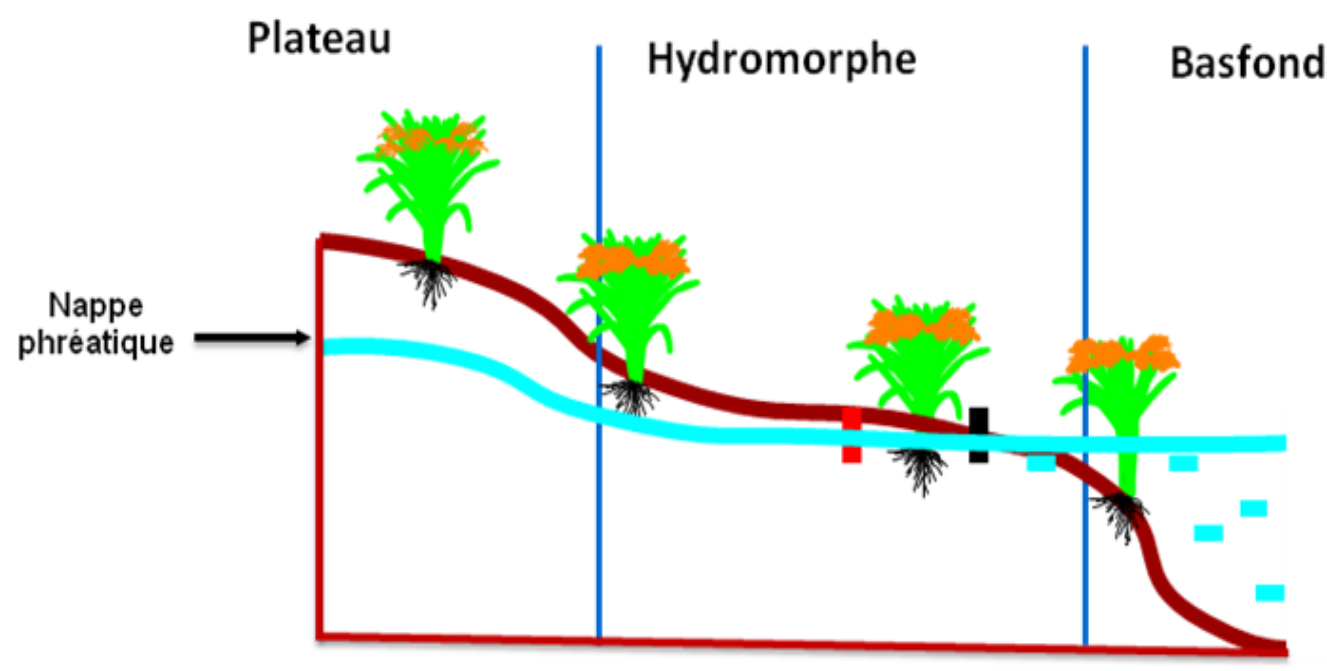

Figure 1 : Représentation des différents écosystèmes de plateau, hydromorphe et bas-fond dans le continuum.

Representation of the various ecosystems, upland, hydromorphe and low land in the continuum.

\section{MATERIEL ET METHODES}

\section{MATERIEL}

L'étude a été réalisé sur le site du Centre du riz pour l'Afrique ou <<Africa Rice >> (ex-ADRAO) dans la vallée de M'Bé à Bouaké. Le matériel végétal est constitué d'anthères provenant de 3 variétés de 0 . sativa de type japonica (WAB 56-50, TAIPEI 309) et indica (BOUAKE 189) et 2 variétés de $O$. glaberrima (CG 14 et CG 20). Les semences de chacune des 5 variétés ont été obtenues de la collection du Département de la Sélection Variétale de <<Africa Rice>>.

\section{METHODES}

\section{Conditions de Semis}

Les cinq variétés ont été semées dans 3 types d'écosystèmes que sont le plateau, l'hydromorphe, et le bas-fond (Figure 2). Les variétés ont été disposées en bloc de FISHER à 3 répétitions sur des parcelles élémentaires de $3 \mathrm{~m}^{2}$. Le défrichage des terrains a été effectué à l'aide d'un motoculteur. Une quantité de
$150 \mathrm{~kg} \mathrm{ha}^{-1}$ d'engrais de fond, NPK 10-18-18, a été appliquée sur chacun des écosystèmes au semis, suivi de $40 \mathrm{~kg} \mathrm{ha}^{-1}$ d'urée 20 jas (période de tallage) et la même quantité 40 j plus tard (période de la montaison des panicules). Un désherbage manuel a été effectué juste avant chaque épandage d'urée. Les données du niveau de la nappe phréatique et de la composition du sol des écosystèmes ont été obtenues avec l'assistance du Département de Pédologie d'<<Africa Rice $>>$.

\section{Prélèvement des panicules}

Les panicules ont été prélevées sur les talles primaires aux stades jeune, intermédiaire et âgé de leur développement, alors qu'elles étaient encore emprisonnées dans les gaines des feuilles paniculaires (dernières feuilles des tiges). Ces 3 stades correspondent respectivement aux distances auriculaires de [7 à 8,8 cm], [8,9 à $10,9 \mathrm{~cm}$ ] (Figure 3A) et [11 à $13 \mathrm{~cm}$ ]. Une fois récoltées, les panicules sont placées au réfrigérateur à $12^{\circ} \mathrm{C}$ pendant $10 \mathrm{j}$. La distance auriculaire est la distance entre le sommet de la gaine paniculaire et la ligule de l'avant dernière feuille. 


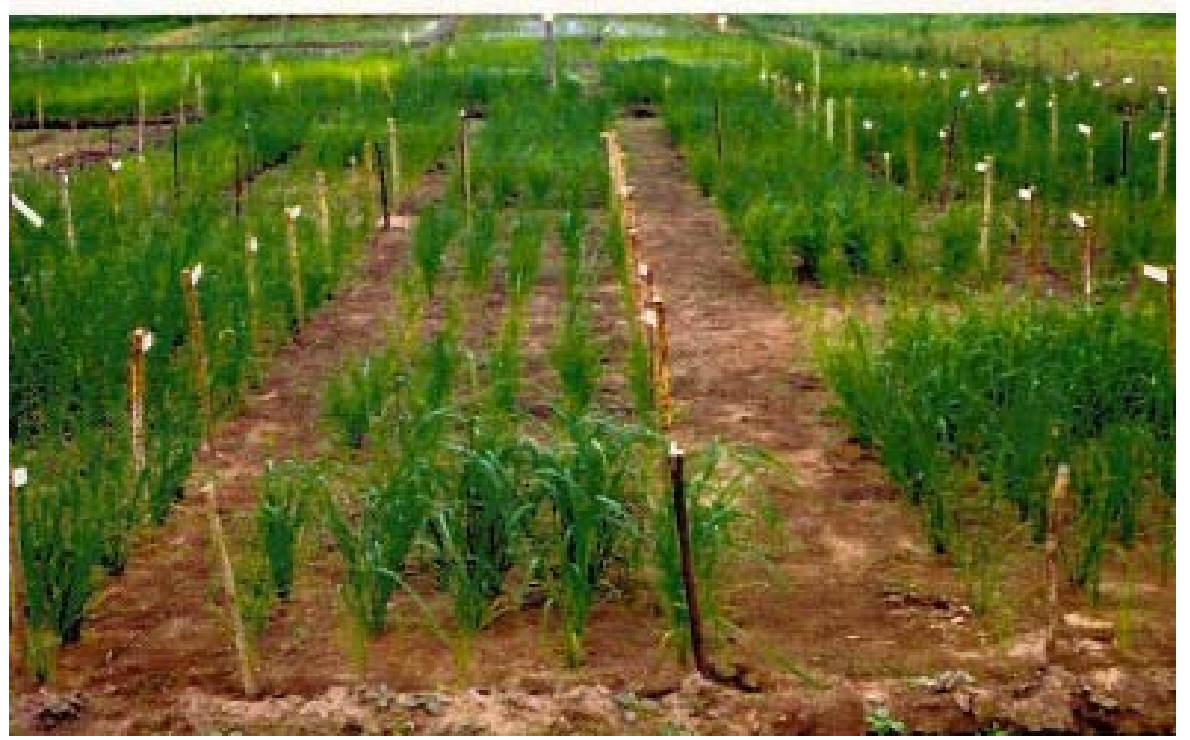

Figure 2 : Les variétés en phase de tallage dans le continuum après 15 jours de semis. Varieties at tillering stage in the continuum 15 days after planting.

\section{Etude cytologique des microspores}

Après le prétraitement au froid, du matériel issu des 3 stades de développement, des observations cytologiques ont été faites en vue d'identifier les épillets contenant le plus de microspores uninucléées tardif reconnus plus favorable à l'androgenèse (Chaleff et Stolarz, 1981 ; Asselin de Beauville, 1976). Les épillets ont été fixés dans de l'alcool acétique $(3: 1)$ pendant 24 heures, puis conservés au réfrigérateur dans de l'alcool $70 \%$. Une fois retirées du réfrigérateur, les anthères ont été isolées à l'aide d'une loupe binoculaire, puis colorées avec l'acétocarmin à $1 \%$ sur une lame et observer microscopique (objectif $20 \mathrm{x}$ ) en vue d'identifier les microspores favorables à l'androgenèse dans les différents types d'épillets (jaunes, Jaune-vert et verts).

\section{Mise en culture}

Les panicules encore enveloppées par leurs gaines sont trempées dans de l'alcool à $70 \%$ pendant une minute puis dans une solution d'hypochlorite de calcium à $0,5 \%$ pendant 20 $\mathrm{mn}$ et rincées immédiatement dans 3 bains successifs d'eau distillée stérile. Les gaines paniculaires ont été otées et les épillets (couleur jaune-vert et transparent) (Figure 3B) ont été disséqués, puis les anthères ensemencées sur le milieu d'induction, à raison de 40 à 50 anthères par flacon. Les observations cytologiques ont montré que ces épillets contenaient beaucoup de microspores uninucléés tardifs. Les flacons ont été ensuite placés à l'obscurité, à $25^{\circ} \mathrm{C}$ pendant 10 à 17 semaines. Le milieu d'induction de cals est constitué du milieu N6 (Chu, 1975) modifié contenant $0,5 \mathrm{mg} \mathrm{l}^{-1}$ de kinétine, $2,5 \mathrm{mg} \mathrm{l}^{-1}$ de 2,4-D, auquel on a ajouté du maltose $50 \mathrm{gl}^{-1}$ et $15 \%$ de lait de coco.

Les cals développés sur les anthères et ayant un diamètre minimum de 2 à $3 \mathrm{~mm}$ sont repiqués sur le milieu de régénération, à raison de 10 cals maximum par flacon, puis placés sous une intensité d'éclairement de 2500 lux, avec une photopériode de $16 \mathrm{~h} \mathrm{j}^{-1}$ et une température de $25^{\circ} \mathrm{C}$. Le milieu de régénération est constitué du milieu MS (Murashige et Skoog, 1962) additionné de $1 \mathrm{mg} \mathrm{l}^{-1} \mathrm{~d}^{\prime} \mathrm{ANA}, 4 \mathrm{mg} \mathrm{l}^{-1}$ kinétine et de $30 \mathrm{~g} \mathrm{l}^{-1}$ de saccharose.

Les plantules vertes régénérées, à partir des cals ayant une taille de $4 \mathrm{~cm}$ au moins, ont été transférées sur un milieu d'enracinement et mises dans la chambre de culture. Les plantules issues du milieu d'enracinement ont été transférées en terre et arrosées avec de l'eau. Le milieu de développement des racines a été composé du milieu de base MS auquel on a ajouté $1 \mathrm{mg} \mathrm{l}^{-1}$ d'ANA, $60 \mathrm{~g} \mathrm{l}^{-1}$ de saccharose, $100 \mathrm{mg} \mathrm{l}^{-1}$ d'acide ascorbique et $2,5 \mathrm{mg} \mathrm{l}^{-1} \mathrm{de}$ MET (Multi Triazole Effect).

Pour l'ensemble des milieux utilisés, le pH a été ajusté à 5,8 à l'aide de $\mathrm{NAOH}$ ou d' $\mathrm{HCl}$ après adjonction du gelrite $\left(2 \mathrm{gl}^{-1}\right)$. Ensuite, les milieux 
ont été chauffés et mis dans des flacons à raison de $10 \mathrm{ml}$ par flacon pour le milieu d'induction, $25 \mathrm{ml}$ par flacon, pour le milieu de régénération et $15 \mathrm{ml}$ par tube pour le milieu d'enracinement. La stérilisation des milieux a été faite à l'autoclave à $116^{\circ} \mathrm{C}$ pendant $30 \mathrm{~min}$.

Les données collectées ont porté sur les pourcentages de cals, sur la base respective de : du nombre d'anthères, de cals obtenus, de plantules vertes, d'albinos, d'haploïdes, de diploïdes et de polyploïdes. Une analyse de variance (test Newman-Keuls) a été appliquée aux données à l'aide de Statitcf, version 5 pour la comparaison des moyennes au seuil de $5 \%$. Les différents paramètres ont été transformés en utilisant la fonction Arc sinus car variant dans les limites de 0 - $100 \%$.

\section{RESULTATS}

\section{AGE DE LAPANICULE ET ECOSYSTEME}

Quelque soit le stade de provenance des panicules, la callogenèse a débuté par un gonflement des anthères qui ont éclaté pour libérer des cals jaune laiteux (Figure 3C et D). Toutefois, le stade de la panicule favorable à l'androgenèse a varié en fonction de l'espèce, du génotype et de l'écosystème (Tableau 1).

En ce qui concerne l'écosystème plateau, chez les glaberrima (CG 14 et CG 20), le stade âgé des panicules a produit plus de cals. Par contre, les cals régénérant des plants verts proviennent du stade jeune de la panicule. Avec les sativa (TAIPEI 309, WAB 56-50 et BOUAKE 189), la meilleure performance pour la production de cals a été obtenue aux stades jeune et intermédiaire de la panicule, mais le taux de production de plants verts a varié d'un génotype à l'autre. En effet, les taux de plants verts ont été semblables pour les 3 stades chez TAIPEI 309, mais, chez WAB 56-50, les meilleurs pourcentages de plants verts ont été observés avec le stade âgé. II n'y a pas eu de régénération de plants verts avec BOUAKE 189. Des plantules non chlorophylliennes ou albinos (Figure 3G) ont également été obtenues. Elles ont été observées chez les deux espèces et aux différents stades de la panicule. Les pourcentages les plus élevés d'albinos ont été observés au stade jeune chez le glaberrima CG 20 (6,4\%), au stade intermédiaire chez le japonica WAB 56-50 $(24,9 \%)$ et au stade âgé chez l'indica BOUAKE $189(19,3 \%)$.
En hydromorphe, le stade jeune est plus favorable, aussi bien à la callogenèse qu'à la régénération de plantules pour les glaberrima (CG 20 et CG 14). Le peu de cals produits aux autres stades chez cette espèce n'a pu régénérer, mais a nécrosé. Chez les sativa (TAIPEI 309 et WAB 56-50), la meilleure performance en production de cals a été obtenue au stade jeune et celle de la régénération au stade inter-médiaire. Le plus important en androgenèses étant la régénération de plants verts, le stade intermédiaire est donc retenu pour les sativa. II n'y a pas eu de production d'albinos chez les glaberrima. Par contre, chez les sativa le plus fort pourcentage a été observé chez WAB 56-50, avec les cals provenant du stade âgé de la panicule.

Pour le bas-fond le stade jeune des panicules est le plus favorable aussi bien pour la production de cals que pour la régénération de plantules des sativa, tout particulièrement chez BOUAKE 189 chez lequel on observe une forte régénération et même plus de cals produits que les glaberrima. Chez ces derniers, le stade jeune a été plus performant aussi bien pour la production de cals que la régénération de plantules. Un pourcentage important d'albinos est observé à tous les stades chez WAB 56-50 plus particulièrement au stage âgé de la panicule (Tableau 1).

\section{GENOTYPE ET ECOSYSTEME}

Au Plateau, les taux de cals les plus élevés ont été obtenus chez les sativa de type japonica, TAIPEI 309 (31,7\%) et WAB 56-50 (6,5\%). Le taux le plus faible a été observé chez les sativa de type indica BOUAKE 189 (0,3\%) même si on n'observe pas de différence significative entre ce dernier et les glaberrima CG $20(2,3 \%)$ et CG 14 (1,8\%). Le japonica TAIPEI 309 (10,4\%) et CG $14(3,1 \%)$ ont régénéré plus de plants verts (Tableau 2). Il a également été observé un taux important d'albinos avec WAB 56-50 $(16,6 \%)$. Tout un bocal de cals transférés peut produire des albinos chez ce génotype (Figure $3 G)$ alors que chez les autres les plants verts et albinos sont produits dans le même bocal.

En hydromorphe les génotypes se comportent de la même manière qu'au plateau pour la production des cals (Tableau 2). Les génotypes les plus productifs de plants verts sont les sativa de type japonica WAB 56-50 (6,3\%) et TAIPEI 309 (5,1\%). Peu de plants sont obtenus avec les glaberrima CG $14(0,7 \%)$ et CG $20(1,7 \%)$. 
Un taux d'albinos important a été observé avec WAB 56-50 (13,5\%).

Au niveau du bas-fond le sativa de type indica BOUAKE $189(0,8 \%)$ a produit plus de cal que les glaberrima, CG $20(0,3 \%)$ et CG $14(0,3 \%)$ chez lesquels on a observé aucune différence significative. Les génotypes les plus productifs en callogenèse restent TAIPEI 309 et WAB 5650. Toutefois le taux de plant verts de BOUAKE 189 (5,9\%) a été significativement supérieur à celui de tous les autres génotypes (Tableau 2).

Les sativa, avec un taux variant entre 0,4 et $21,3 \%$ (Tableau 3), ont produit plus de cals que les glaberrima, dont les taux ont atteint un maximum de $1,4 \%$, tous écosystèmes confondus. En effet comme l'illustre la figure $3 \mathrm{C}$ et $D$ on observe plus de cals dans les bocaux du sativa TAIPEI $309(21,3 \%)$ que du glaberrima CG 20 (1,4\%), les meilleurs génotypes en callogenèse chez chacune des espèces. Tout comme en callogenèse le plus productif en régénération est TAIPEI $309(6,1 \%)$ pour les sativa. Par contre, chez les glaberrima on a CG 14 (1\%). Chez TAIPEI 309, tous les cals d'un bocal peuvent générer des points verts qui ensuite se développent en plants verts (Figure 3E \& F). Tous les plants verts transférés quelque soit leur origine ont développé des racines sur le milieu d'enracinement comme le montre la figure $3 \mathrm{H}$ chez CG 14.

Tableau 1 : Moyenne des pourcentages de cals et de plantules à 3 stades croissance de la panicule pour 3 écosystèmes

Average percentage of callus, plantlets at 3 stages of panicle development for the 3 ecosystems

\begin{tabular}{|c|c|c|c|c|c|c|c|c|c|c|}
\hline \multirow{2}{*}{$\begin{array}{l}\text { Stade de } \\
\text { Panicule }\end{array}$} & \multirow[b]{2}{*}{ Génotype } & \multicolumn{3}{|c|}{ Plateau } & \multicolumn{3}{|c|}{ Hydromorphe } & \multicolumn{3}{|c|}{ Bas-fond } \\
\hline & & Cals (\%) & Plvt & Alb & Cals (\%) & Plvt & Alb & $\begin{array}{l}\text { Cals } \\
(\%)\end{array}$ & Plvt & Alb \\
\hline \multirow[t]{5}{*}{ Jeune } & CG 14 & $3,7 c$ & $9,2 b$ & Oc & $0,7 c$ & $2,1 b$ & $0 c$ & $0,9 d$ & $0 \mathrm{~b}$ & $0 c$ \\
\hline & CG 20 & $0,9 c$ & $3,1 c$ & $6,4 c$ & $1,2 \mathrm{c}$ & $4,3 b$ & $0 c$ & $1 d$ & $0 b$ & $0 c$ \\
\hline & BOUAKE 189 & $0,1 \mathrm{c}$ & $0 c$ & $0 c$ & $0 c$ & $\mathrm{Ob}$ & $0 c$ & $1,5 d$ & $13,1 \mathrm{a}$ & $0 c$ \\
\hline & WAB 56-50 & $7,1 b$ & $3,8 c$ & $10,5 b c$ & $9,6 b$ & $5,3 a b$ & $6,2 b c$ & $4,2 d c$ & $2,5 b$ & $14,3 a b$ \\
\hline & TAIPEI 309 & $34,9 a$ & $14,6 a$ & $7,2 \mathrm{c}$ & $18,7 a$ & $8,7 a$ & 3,3 & $22,8 \mathrm{a}$ & $3,3 b$ & $1,6 c$ \\
\hline \multirow[t]{5}{*}{ Intermédiaire } & CG 14 & $0 c$ & $0 c$ & oc & $0,2 c$ & $\mathrm{Ob}$ & Oc & Od & $0 \mathrm{~b}$ & oc \\
\hline & CG 20 & $0 c$ & $0 c$ & $0 c$ & $3,1 \mathrm{c}$ & $\mathrm{Ob}$ & $0 c$ & od & $\mathrm{Ob}$ & Oc \\
\hline & BOUAKE 189 & $0,8 c$ & oc & $0 c$ & oc & $\mathrm{Ob}$ & $0 c$ & $0,8 \mathrm{~d}$ & $4,7 b$ & $2,4 c$ \\
\hline & WAB 56-50 & $7,3 b$ & $3,2 \mathrm{c}$ & $24,9 a$ & $8 b$ & $9,2 \mathrm{a}$ & $10,9 b$ & $4,5 \mathrm{dc}$ & $1,8 b$ & $11,6 a b$ \\
\hline & TAIPEI 309 & $31,8 a$ & $12,4 a$ & $5,3 c$ & $17 a$ & $4,4 b$ & $3,3 c$ & $11,2 b$ & $2,6 b$ & $2,1 \mathrm{c}$ \\
\hline \multirow[t]{5}{*}{ Agé } & CG 14 & $1,6 c$ & $0 c$ & $0 c$ & $0,6 c$ & $\mathrm{Ob}$ & $0 c$ & Od & $\mathrm{Ob}$ & $0 c$ \\
\hline & CG 20 & $5,9 c$ & $0 c$ & $4,9 c$ & $0,7 c$ & $0 \mathrm{~b}$ & $0 c$ & Od & $0 b$ & $0 c$ \\
\hline & BOUAKE 189 & $0,1 \mathrm{c}$ & $0 c$ & $19,3 a$ & $0 c$ & $\mathrm{Ob}$ & $0 c$ & Od & $\mathrm{Ob}$ & $0 c$ \\
\hline & WAB 56-50 & $5,1 c$ & $4,9 c$ & $14,5 b$ & $9,2 b$ & $4,3 b$ & $23,5 a$ & $7 c$ & $2,7 b$ & $18,7 a$ \\
\hline & TAIPEI 309 & $28,3 a b$ & $4,2 \mathrm{c}$ & $3,2 \mathrm{c}$ & $14,2 \mathrm{ab}$ & $2,2 b$ & $5,4 b c$ & $12,5 b$ & $2,1 b$ & $5,1 b$ \\
\hline
\end{tabular}

Les chiffres suivis par la même lettre ne sont pas significativement différents au seuil alpha égal à $5 \%$. Plvt : plantules vertes ; Alb : albinos

Numbers followed by the same letter are not significantly different at alpha equal $5 \%$.

Tableau 2 : Moyenne des pourcentages de cals et de plantes dans l'interaction génotypes-écosystème Average percentage of the callus and plantlets in the interaction genotypes-ecosystem

\begin{tabular}{llccccccccc}
\hline \multirow{2}{*}{ Espèce } & \multirow{2}{*}{ Génotype } & \multicolumn{3}{c}{ Plateau } & \multicolumn{3}{c}{ Hydromorphe } & \multicolumn{3}{c}{ Bas-fond } \\
\cline { 2 - 10 } & & Cals (\%) & Plvt & Alb & Cals (\%) & Plvt & Alb & Cals (\%) & Plvt & Alb \\
\hline \multirow{2}{*}{ Glaberrima } & CG 14 & $1,8 \mathrm{c}$ & $3,1 \mathrm{ab}$ & $0 \mathrm{c}$ & $0,5 \mathrm{c}$ & $0,7 \mathrm{~b}$ & $0 \mathrm{~b}$ & $0,3 \mathrm{c}$ & $0 \mathrm{c}$ & $0 \mathrm{c}$ \\
& CG 20 & $2,3 \mathrm{c}$ & $1 \mathrm{~b}$ & $3,8 \mathrm{c}$ & $1,7 \mathrm{c}$ & $1,4 \mathrm{~b}$ & $0 \mathrm{~b}$ & $0,3 \mathrm{c}$ & $0 \mathrm{c}$ & $0 \mathrm{c}$ \\
\hline \multirow{2}{*}{ Sativa } & BOUAKE 189 & $0,3 \mathrm{c}$ & $0 \mathrm{~b}$ & $6,4 \mathrm{~b}$ & $0 \mathrm{c}$ & $0 \mathrm{~b}$ & $0 \mathrm{~b}$ & $0,8 \mathrm{c}$ & $5,9 \mathrm{a}$ & $0,8 \mathrm{c}$ \\
& WAB 56-50 & $6,5 \mathrm{~b}$ & $4 \mathrm{~b}$ & $16,6 \mathrm{a}$ & $8,9 \mathrm{~b}$ & $6,3 \mathrm{a}$ & $13,5 \mathrm{a}$ & $5,2 \mathrm{~b}$ & $2,3 \mathrm{~b}$ & $14,9 \mathrm{a}$ \\
& TAIPEI 309 & $31,7 \mathrm{a}$ & $10,4 \mathrm{a}$ & $5,2 \mathrm{~b}$ & $16,6 \mathrm{a}$ & $5,1 \mathrm{a}$ & $4, \mathrm{ab}$ & $15,5 \mathrm{a}$ & $2,7 \mathrm{~b}$ & $2,9 \mathrm{~b}$ \\
\hline
\end{tabular}

Les chiffres suivis par la même lettre ne sont pas significativement différents au seuil alpha égal à $5 \%$. Plvt : plantules vertes ; Alb albinos

Numbers followed by the same letter are not significantly different at alpha equal $5 \%$. 
Tableau 3 : Influence du génotype sur la production de cals et de plantules à partir des anthères. Influence of the genotype on callus and plantlets production from anthers.

\begin{tabular}{lccc}
\hline Matériel Végétal & $\%$ Cals & $\%$ Plvt & $\% \mathrm{Alb}$ \\
\hline O. Glaberrima & 1,2 & 1 & 0,6 \\
CG 14 & $0,9 \mathrm{c}$ & $1,3 \mathrm{~b}$ & $0 \mathrm{c}$ \\
CG 20 & $1,4 \mathrm{c}$ & $0,8 \mathrm{~b}$ & $1,3 \mathrm{c}$ \\
O. Sativa & 9,5 & 4,1 & 7,1 \\
BOUAKE 189 (indica) & $0,4 \mathrm{c}$ & $2 \mathrm{~b}$ & $2,4 \mathrm{~b}$ \\
WAB 56-50 (Japonica) & $6,9 \mathrm{~b}$ & $4,2 \mathrm{a}$ & $15 \mathrm{a}$ \\
TAIPEI 309 (Japonica) & $21,3 \mathrm{a}$ & $6,1 \mathrm{a}$ & $4 \mathrm{~b}$ \\
\hline
\end{tabular}

Les moyennes suivies par la même lettre ne sont pas significativement différents au seuil alpha égal à $5 \%$. Plvt : plantules vertes; Alb : albinos

Numbers followed by the same letter are not significantly different at alpha equal $5 \%$.

\section{Ecosystème}

Lorsqu'on considère l'ensemble des génotypes, les analyses montrent que le bas-fond $(4,4 \%)$ et l'hydromorphe $(5,5 \%)$ ont eu des taux de cals statistiquement comparables (Tableau 4) et différents de ceux du Plateau (8,5\%). La production de cals décroît dans le sens plateau, hydromorphe et bas-fond. La même tendance a été observée pour la régénération de plants verts pour laquelle le bas-fond $(2,2 \%)$ et l'hydromorphe $(2,7 \%)$ ont eu les taux faibles, par rapport au plateau (3,7\%). II n'y a pas eu de différence significative pour le niveau de ploïdie aussi bien pour les haploïdes, diploïdes et polyploïdes entre les 3 écosystèmes. Sous serre, les haploïdes se sont distingués des autres plants par leur très petites taille et une panicule verte totalement stérile à maturité (Figure I(1) et J(1)). Les polyploïdes se sont différenciés par leur grande taille et leur panicules de taille anormale et fertiles, à moins de 50\% (Figure I(2) et J(2)), par rapport aux diploïdes spontanés (Figure I(3) et $\mathrm{J}(3)$ ) semblables à la plante mère.

Tableau 4 : Moyenne des pourcentages de cals, plantules et du niveau de ploïdie pour les 3 écosystèmes.

Average percentage of callus, plantlets and the ploiddie level for the 3 ecosystems.

\begin{tabular}{lcccccc}
\hline Ecosystème & cal & Plvt & Alb & Haploïde & Diploïde & Polyploïde \\
\hline Plateau & $8,5 \mathrm{a}$ & $3,7 \mathrm{a}$ & $6,4 \mathrm{a}$ & 76,4 & 18,5 & 5,1 \\
Hydromorphe & $5,5 \mathrm{~b}$ & $2,7 \mathrm{~b}$ & $3,5 \mathrm{~b}$ & 70,70 & 22,20 & 7,10 \\
Bas-fond & $4,4 \mathrm{~b}$ & $2,2 \mathrm{~b}$ & $3,7 \mathrm{~b}$ & 72,80 & 22,30 & 4,90
\end{tabular}

Les moyennes suivies par la même lettre ne sont pas significativement différents au seuil alpha égal à $5 \%$. Plvt : plantules vertes ; Alb : albinos

Numbers followed by the same letter are not significantly different at alpha equal $5 \%$. 


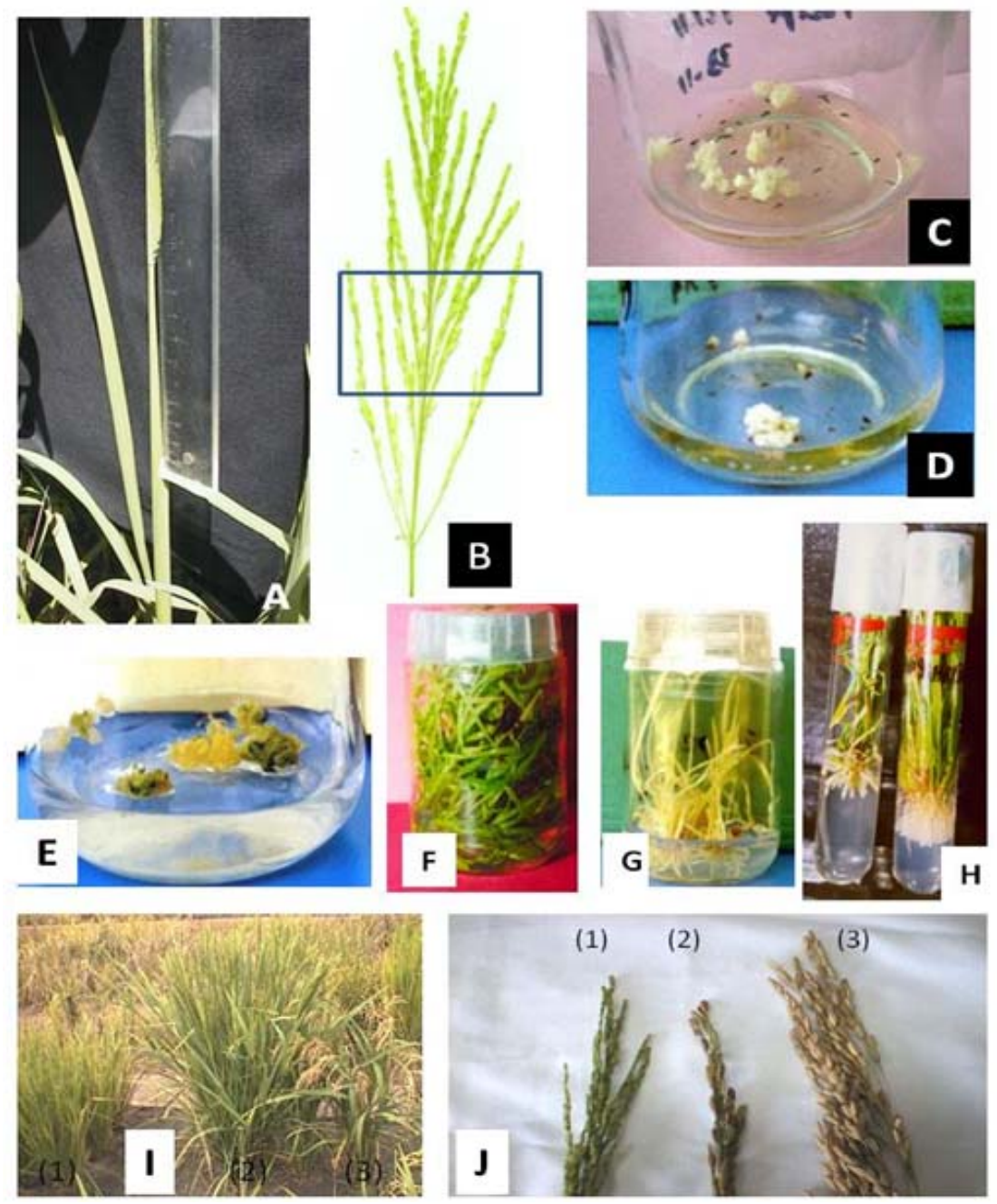

Figure 3 : Panicule au champ avec distance auriculaire de $9 \mathrm{~cm}(\mathrm{~A})$, épillets jaune-verts sélectionnés pour la mise en culture (B), cals de TAIPEI 309 (O. sativa)(C), cals de CG 14 (O. glaberrima) (D); point verts de TAIPEI $309(E)$, plantules vertes de TAIPEI 309 sur milieu de régénération (F), CG 14 sur milieu d'enracinement $(H)$, plantules albinos de WAB 56-50 (G)plante et panicule haploïde (1) diploïde (2) et polyploïde (3) (I \& J).

Panicle in the field with $9 \mathrm{~cm}$ auricular distance (A), spikes yellow-green targeted for anther culture (B), TAIPEI 309 callus (O. sativa) (C), CG 14 callus (O. glaberrima) (D), Green points of TAIPEI 309 (E), TAIPEI 309 green plantlets on regeneration (F), CG 14 on rooting medium (H), Albino plants of WAB 56-50 (G), Haploid (1), diploid (2) and plolyploids (3) plants and panicles (I $\& J)$.

\section{DISCUSSION}

Les études précédentes, en ce qui concerne l'environnement des plantes-mères, se rapportent au climat et à la fertilité d'un type d'écosystème bien défini (Zhenhua, 1992 ; Zapata, 1985). Car, pour limiter les besoins en eau, les bas-fonds sont préférentiellement choisis pour les plantes candidates à la culture d'anthères. Nos résultats montrent pourtant que cet écosystème n'est pas favorable à l'andro- genèse pour tous les génotypes. En effet, il y a eu une forte interaction génotypes-écosystèmes. Chaque génotype utilisé a répondu en fonction de son environnement naturel. Les glaberrima (CG 14 et CG 20) et les sativa de type japonica (TAIPEI 309 et WAB 56-50) ont mieux répondu avec les panicules provenant du plateau qui est leur écosystème habituel de croissance. Le sativa de type indica (BOUAKE 189) a été plus performant avec les panicules provenant du bas-fond, son habitat naturel. Par 
conséquent, le taux élevé de cals et aussi de plants verts obtenus au plateau est le fait du nombre relativement important de génotypes ayant pour habitat cet écosystème. Toutefois, bien que leur écosystème naturel soit le plateau, WAB 56-50 et TAIPEI 309 ont été également performant en hydromorphe montrant qu'ils ont une bonne capacité d'adaptation. La composition des sols (Tableau 5) et le niveau de la nappe phréatique (Figure 4) différent d'un écosystème à un autre, il est donc possible que chacun de ces écosystèmes ait influencé l'état physiologique des plantes qui selon Zhenhua (1992) peut affecter, de façon significative, la callogenèse. Toutefois, le mécanisme par lequel les écosystèmes affectent la production de cals et, par voie de conséquence, des plantules vertes reste à élucider.

En plus de la forte interaction génotypeécosystème, sur le rendement en androgenèse, un effet génotype significatif a été aussi observé. L'influence prédominante du génotype en androgenèse chez le riz a été largement décrite (Faruque et al., 2004 ; Yan et al., 1996 ; Bagheri et Jelodar, 2008). II a été rapporté que chez les sativa, les génotypes japonica répondent mieux en androgenèse que les indica (Shen et al., 1982). Cette observation est en accord avec nos résultats, qui, par ailleurs ont montré qu'il y a également un effet génotype chez les glaberrima, où CG 20 a produit moins de plants verts par rapport à CG 14. Cet effet génotype chez les glaberrima a été aussi observé par Gueye et Ndir (2010). Les travaux de Woo et al. (1983) ont rapporté une callogenèse relativement élevé, supérieure à la nôtre, avec des variétés de glaberrima non identifiés. Toutefois, le taux de régénération est comparable à nos résultats. Mais, les taux très élevés de diploïdes spontanés, plus du double des haploïdes, suggère qu'une partie des cals pourrait provenir des parois des anthères. Car, dans la plupart des études sur l'andro-genèse du riz, le taux d'haploïdes est de loin supérieur à celui des diploïdes spontanés.

Mercy et al. (1986), ont étudié, en bas-fond, l'effet du stage de développement sur l'induction de cals d'anthères chez deux variétés japonica, dont TAIPEI 309, en considérant comme marqueur morphologique la distance auriculaire entre 4 - 12,5 cm. Ils ont montré que le stade de développement a influençé très peu la production des cals, car des distances auriculaires de 8 $\mathrm{cm}$ et $12 \mathrm{~cm}$ chez TAIPEI 309 ont eu des performances comparables. Ces observations contredisent nos résultats, qui ont montré une différence dans la production des cals chez TAIPEI 309, entre les stades de développement de la panicule. Cette différence avec les résultats de Mercy et al. (1986) pourrait être due aux différences entre milieux de culture des anthères.

La production de plantules albinos est un phénomène inhérent à la culture d'anthères des céréales (Clapham, 1977). Chez le riz, la fréquence d'albinos a varié de $10 \%$ (Wang et al., 1977 ; Genovesi and Magil 1979) à $100 \%$ (Agrawal et Agrawal 1991). La production d'albinos semble avoir été influencée par les facteurs tels la température d'incubation, la lumière, les milieux de culture, le stade de développement des panicules et l'âge des cals transférés (Shahnewaz et Bari, 2004 ; Wang et al., 1977). De plus, la forte production d'albinos, quelque soitl'écosystème et le stade de la panicule observé chez le génotype WAB 56-50, suggère que l'albinisme pourrait avoir une base génétique. Ce qui confirme les observations de Masumi (2004) sur le riz et de Tuvessonetal. (1989) sur le blé.

Tableau 5 : Données de l'analyse du sol des trois écosystèmes.

Data of the soil analysis for the three ecosystems.

\begin{tabular}{|c|c|c|c|c|c|c|c|c|}
\hline Ecosystème & $\begin{array}{l}\text { Arg + } \\
\operatorname{Lim} \%\end{array}$ & $\begin{array}{l}\mathrm{N} \text { total } \\
(\mathrm{ppm})\end{array}$ & $\begin{array}{l}\text { P total } \\
(\mathrm{ppm})\end{array}$ & $\begin{array}{l}\text { SBE } \\
\mathrm{me} / 100 \mathrm{~g}\end{array}$ & $\begin{array}{l}\mathrm{CEC} \\
\mathrm{me} / 100 \mathrm{~g}\end{array}$ & $\begin{array}{l}\mathrm{BE} \\
\text { sat \% }\end{array}$ & Fertilité & $\begin{array}{l}\text { Conductivité } \\
\mathrm{ms} / \mathrm{cm}\end{array}$ \\
\hline Plateau & $38 \mathrm{a}$ & $500 c$ & $90 \mathrm{~b}$ & $2,98 \mathrm{c}$ & $3,42 b$ & $87 \mathrm{~b}$ & $0,23 \mathrm{c}$ & $0,7 \mathrm{a}$ \\
\hline Hydromorphe & $38 \mathrm{a}$ & $700 \mathrm{a}$ & $125 \mathrm{a}$ & $8,99 \mathrm{a}$ & 7,58ab & $119 a$ & $2,12 \mathrm{a}$ & $0,1 \mathrm{~b}$ \\
\hline Bas-fond & $39 a$ & $600 \mathrm{~b}$ & $5 c$ & $6,89 \mathrm{~b}$ & $8,41 \mathrm{a}$ & $82 b$ & $1,21 \mathrm{~b}$ & $0,06 \mathrm{c}$ \\
\hline
\end{tabular}

Les chiffres suivis par la même lettre ne sont pas significativement différentes au seuil d'alpha égal à $5 \%$.

The numbers followed by a letter are not significantly different at alpha equal $5 \%$.

SES: somme des bases ( $\mathrm{Ca}, \mathrm{Mg}, \mathrm{K}, \mathrm{Na}$ ) échangeables ; CEC : capacité d'échange cationique ; BE : taux de saturation; Fertilité : $(\mathrm{SBE})^{2} / \mathrm{Arg}+\mathrm{Lim}$. Condition climatique moyenne: pluviométrie $=134,5 \mathrm{~mm}$; température : $25,3^{\circ} \mathrm{C} ;$ rayonnement $=0,2 \mathrm{kw} / \mathrm{m}^{2} ;$ vitesse $\mathrm{du}$ vent $=2 \mathrm{~m} / \mathrm{s}$. 


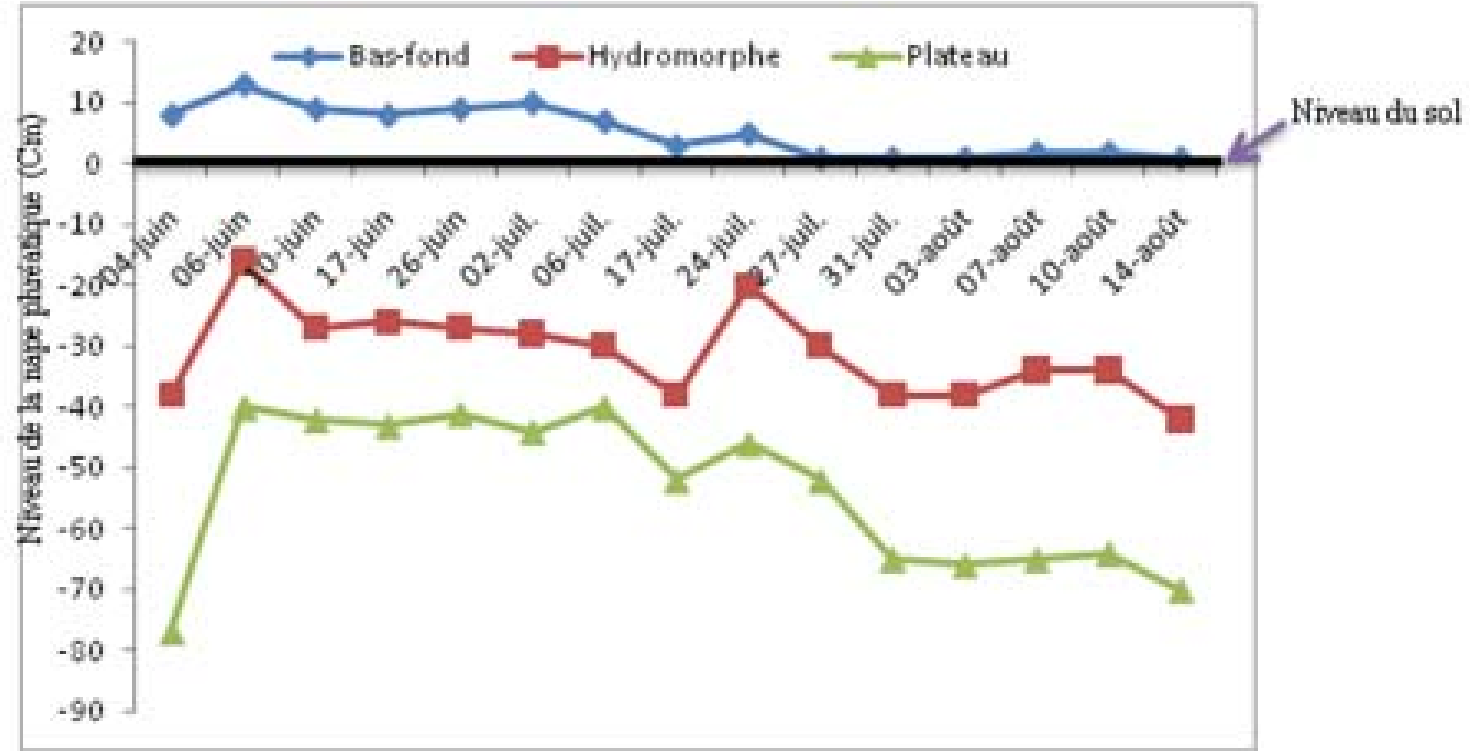

Figure 4 : Niveau moyen de la nappe phréatique dans les différents écosystèmes pendant la conduite des essais.

The average level of the groundwater in the various ecosystems during the experimentation.

\section{CONCLUSION}

En androgenèse, l'écosystème plateau a été plus favorable, par ordre d'importance, aux japonica (TAIPEI 309 et WAB 56-50) et glaberrima (CG 14 et CG 20). La zone hydromorphe convient uniquement aux japonica (TAIPEI 309 et WAB 56-50). L'écosystème basfond a été plus favorable au sativa de type indica (BOUAKE 189). Ainsi, l'écosystème agit sur le rendement en androgenèse et suggère le maintien des génotypes dans leur éco-système habituel pour une bonne androgenèse. En effet, les sativa à fond génétique indica ont été performants dans les bas-fonds et ceux de fond génétique glaberrima et japonica sur les plateaux. L'étude a également révélé que l'âge des panicules est important et que, quelle que soit l'espèce, les pannicules doivent être relativement jeunes.

\section{REMERCIEMENTS}

Nous sommes infiniment reconnaissants à Toshio Murashige pour ses conseils et son aide pendant la mise en place de l'unité de culture in vitro à <<AfricaRice >> Nos remerciements vont à la Banque Africaine de Développement (BAD) qui a financé, en partie, cette étude. En outre, ce travail n'aurait pu voir le jour sans le soutien financier de la Fondation Rockefeller que nous remercions sincèrement.

\section{REFERENCES}

Agrawal G. K. and V. P. Agrawal. 1991. Study on haploid and diploid plants of cold tolerant rice cultivar Jumli Marshi, Biotech. Biodiv. Lett. 1 : 10 - 11.

Angladette A. 1966. Le riz, Maisonneuve et Larose, Paris, $930 \mathrm{p}$.

Asselin de Beauville M. 1976. Androgenèse in vitro chez Oryza sativa (variété Cigalon). Argon. Trop. $31: 51$ - 57

Bagheri N. and .N B. Jeldar. 2008. Combining ability and heritability of callus induction and green-plant regeneration in rice anther culture. Biotech. 7 (2) : 87 - 292.

Chaleff R. S. and A. Stolarz. 1981. Factors influencing the frequency of callus formation among culture rice (Oryza sativa L.) anthers. Physiol. Plant. 51 : 201 - 206.

Chu C. C. Wang C. S. Hsu K. C. Yin C. Y. and F. B. Chu. 1975. Etablishement of efficient medium for anther culture of rice through comparative experiments sources. Sci. Sin. $18: 659-668$. 
Chung G. 1992. Anther culture for rice improvement in Korea. In anther culture for rice breeder. Edited by Zheng Kagle and Toshio Murashige. pp 8 - 37.

Clapham D. 1977. Haploid induction in cereals. In : J. Reinert. Fundamental aspects of plant cell, tissue, and organ culture. SpringerVerlag, Berlin, Heiderberg, New york : pp 279 - 298.

Constance N., Chowdhury A. K., Tyagi N. and S. C. Maheshwari. 1993. Effect of L. proline and L. tryptophane on somatic embryogenesis plantlet regeneration of rice (O. sativa L.). Plant cell. Tissue and organ culture. 32 (3) : 357 - 361.

Cornejo-Maritin M. J. and E. Primo-Millo. 1981. Anther and pollen grain culture of rice (Oryza sativa L.). Euphytica. (3) 30 : 81 - 89.

Faruque O. M. Farzana T. Seraj I. Z. Sarker R. H. and $A$. A. Khatun. 2004. Variations in green plant regeneration response from anthers of indica rice and their hybrids with japonica cv. Taipei 309. Plant Cell, Tissue and Organ Culture. 54 (3) : 191 - 195

Jähne A. and H. Lörz. 1995. Cereal microspore culture. Plant Sci. 109 : 1 - 12

Genovesi A. D. and C. W. Magill. 1979. Improved rate of callus and green plant production from rice anther culture following cold shock. Crop Sci. 19 : 662 - 664.

Guèye T. and K. N. Ndir. 2010. In vitro production of double haploid plants from two rice species (Oryza sativa L. and Oryza glaberrima Steudt.) for the rapid development of new breeding material. Scientific Research and Essays. 5(7) : 709 - 713.

Huang H.S. Ling T. H. Tseng P. L. Shien Y. L. And P. Shi. 1978. In proceeding of the symposium on plant tissue culture. Beijing, China : Science Press : PP 244 - 246.

Masumi Y. 2004. Heterogeneous plastid genomes in anther culture-derived albino rice plants. Euphytica. $123: 67$ - 74.

Mercy S. T. and F. J. Zapata. 1986. Effect of pollen development stage on callus induction and its relation to auricule distance in tow rice varieties. International Rice Research Newsletter $11: 4$ Monty P. J. Dingkuhn M. Aluko G. K. and S. Mande. 1997. Interspecific Oryza sativa L. X O. glaberrima S. pro- genies in upland rice improvement. Euphytica. 92 : 237 - 246.

Murashige T. and K. Skoog. 1962. A revised medium for rapid growth and bioassays with tabacco tissue cultures. Physiol. Plant.15 : 473 - 497.

Niizeki H. and K. Oono. 1968. Induction of haploid rice plants from anther culture. Proc. Japan Acad.44 : 554 - 557.

Quimio C. A. and F. J. Zapata. 1990. Diallel analysis of callus induction and green plant regeneration in rice anther culture. Crop Science 30(1) : 188 - 192.

Shahnewaz S. and M. A. Bari. 2004. Effect of Concentration of sucrose on the frequency of callus induction and plant regeneration in anther culture of rice (Oryza sativa L.). Plant Tissue Cult. 14 (1) : 37 - 43.

Shen J. H. Li M. F. Chen Y. Q. and Z. H. Zhang. 1982. Breeding by anther culture in rice varieties improveement. Sci. Agricult. Sin. $2: 15-19$.

Torrizo L. B. and F. J. Zapata. 1986. Anther culture in rice: the effect of abscissic acid on plant regeneration. Plant Cell Rep. 5 : 136 - 139.

Tuvesson I. K. D. Pedersen S. and S. B. Andersen. 1989. Nuclear genes affecting albinism in wheat (Triticum aestivum L.) anther culture. Theor. Appl. Genet. $78: 879$ - 883.

Wang C. C, Sun C. S. and C. A. Chu. 1977. An effect of culture factors in vitro on the production of albino pollen-plants of rice. Acta. Bot. Sin. 19 : 190 - 198.

Woo S. K. S. and C. Wong. 1983. Anther culture of pollen plants derived from cross Oryza sativa L. x O. glaberrima Steud. Bot. Bull, Acad. Sin. $24: 53$ - 58.

Yan J. Xue Q, and J. Zhu. 1996. Genetic studies of anther culture ability in rice (Oryza sativa). Plant Cell Tissue and Organ Culture 45 : $253-258$.

Zapata F. J. Adelmita R. R. Torrizo L. B. and L. B. Novero. 1985. Development of cold-tolerant lines through anther culture. Rice genetic. Pp 773 - 780.

Zhenhua Z. 1992. Anther culture for rice breeding at SAS. In anther culture for rice breeder. Edited by Zheng Kagle and Toshio Murashige. pp 8 - 37. 